[Agr. Biol. Chem., Vol. 31, No. 1, p. 73 76, 1967]

\title{
Inhibition of Yeast Growth by Methionine
}

\section{Part I. Nature of the Inhibition}

\author{
By Toshio Takahashi, Yoshitsugu Fujn and Hajime Takahashi
}

Department of Agricultural Chemistry, Faculty of Agriculture, Tohoku University, Sendai

Received August 2, 1966

\begin{abstract}
Growth of an adenine-requiring yeast and a baker's yeast was markedly inhibited by the addition of methionine to culture media. The inhibition was reversed by adenine or more clearly by adenine plus glucose supplements. The inhibition by methionine of the adenine-requiring yeast was more pronounced when the media contained relatively low levels of adenine. Further addition of adenine to the media reversed the inhibition. It appears, therefore, that methionine is a competitive inhibitor of adenine for growth of the yeast cells.
\end{abstract}

\section{INTRODUCTION}

Schlenk and DePalma ${ }^{1 /}$ have reported an accumulation of S-adenosylmethionine in yeast cells which were grown in a medium containing large quantities of methionine. It was indicated in their experiment that, when the yeast was cultivated with methionine, the growth was partially inhibited. Similar observation was made by Kuraishi and Uemura. $^{2)}$ The recent paper by Dalal, Gots and Gots ${ }^{31}$ has shown, on the contrary, that growth of Salmonella typhimurium mutants was inhibited by adenine which was reversed by methionine. These findings led the authors to re-examine the effect of methionine in yeast cells more precisely with special reference to biosynthesis and utilization of adenine compounds. The present paper deals with growth inhibition in yeast cells by methionine and the conditions of its reversal.

1) F. Schlenk and R. E. DePalma, J. Biol. Chem., 229, 1037 (1957).

2) H. Kuraishi and T. Uemura, Amino Acids, 4 95 (1961).

3) F. R. Dalal, R. E. Gots and J. S. Gots, J. Bacteriol., $\mathbf{9 1}, 507$ (1966).

\section{MATERIALS AND METHODS}

Strain and Cultural Conditions. Two strains of yeast were used in this study. One is an adeninerequiring mutant of Saccharomyces cerevisiae and another is a baker's yast (Sankyo) which does not require adenine for its growth. Both strains were from stock cultures of this laboratory. The former strain was originally isolated by Lindegren and additionally requires arginine for growth. Yeasts were precultured at $30^{\circ} \mathrm{C}$ in a medium ( $\mathrm{pH} \mathrm{5.6)}$ consisted of $2 \%$ of glucose, $0.2 \%$ of yeast extract, $0.1 \%$ each of $\left(\mathrm{NH}_{4}\right)_{2} \mathrm{SO}_{4}$ and $\mathrm{KH}_{2} \mathrm{PO}_{4}, 0.05 \%$ of $\mathrm{KCl}, 0.05 \%$ of $\mathrm{MgSO}_{4} \cdot 7 \mathrm{H}_{2} \mathrm{O}$ and $0.001 \%$ of $\mathrm{FeSO}_{4} \cdot 7 \mathrm{H}_{2} \mathrm{O}$. Adenine sulfate $(0.005 \%)$ and L-arginine hydrochloride $(0.02 \%)$ were supplemented to the above medium for the growth of the adenine-requiring yeast. After incubation for 24 hours, cells were washed three times with water and were inoculated to $100 \mathrm{ml}$ of a synthetic medium at concentrations of $10^{4}$ cells $/ \mathrm{mL}$. The synthetic medium was a modification of that described by Kuraishid) and had the following composition; glucose $10.0 \mathrm{~g},\left(\mathrm{NH}_{4}\right)_{2} \mathrm{SO}_{4} 1.25 \mathrm{~g}, \mathrm{Na}_{2} \mathrm{HPO}_{4}$. $2 \mathrm{H}_{2} \mathrm{O} 2.93 \mathrm{~g}$, citric acid $1.78 \mathrm{~g}, \mathrm{CaCl}_{2} \cdot 2 \mathrm{H}_{2} \mathrm{O} 0.1 \mathrm{~g}$, $\mathrm{KCl} 0.6 \mathrm{~g}, \mathrm{MgSO}_{4} \cdot 7 \mathrm{H}_{2} \mathrm{O} 0.5 \mathrm{~g}, \mathrm{H}_{3} \mathrm{BO}_{3} 500 \mu \mathrm{g}, \mathrm{CuSO}_{4}$. $5 \mathrm{H}_{2} \mathrm{O} 40 \mu \mathrm{g}$, KI $100 \mu \mathrm{g}, \mathrm{FeCl}_{3} \cdot 6 \mathrm{H}_{2} \mathrm{O} 200 \mu \mathrm{g}, \mathrm{MnSO}_{4}$. $\mathrm{H}_{2} \mathrm{O} 400 \mu \mathrm{g}, \quad \mathrm{Na}_{2} \mathrm{MoO}_{4} \cdot 2 \mathrm{H}_{2} \mathrm{O} 200 \mu \mathrm{g}, \quad \mathrm{ZnSO}_{4} \cdot 7 \mathrm{H}_{2} \mathrm{O}$

4) H. Kuraishi, Sci. Rep. Tohoku Univ., 4 Ser., Biology, 25, 247 (1959). 
$400 \mu \mathrm{g}$, inositol $2 \mathrm{mg}$, Ca-pantothenate $200 \mu \mathrm{g}$, biotin $2 \mu \mathrm{g}$, pyridoxine hydrochloride $400 \mu \mathrm{g}$ and thiamine hydrochloride $400 \mu \mathrm{g}$ per liter and $\mathrm{pH} 5.6$. For the adenine-requiring strain, $200 \mathrm{mg}$ of L-arginine hydrochloride and, unless otherwise stated, $50 \mathrm{mg}$ (0.124 mmole) of adenine sulfate were added per liter of the above medium. The effect of methionine was examined with the media supplemented with various amounts of DL-methionine. All chemicals were of commercial preparations of reagent grade. The cultures were conducted with shaking at $30^{\circ} \mathrm{C}$.

Determination of Growth. Growth of the yeast cells was followed by optical densities with a Hitachi FPW-4 colorimeter equipped with a 66 filter $(660 \mathrm{~m} \mu)$. One O.D. unit was equivalent to approximately $3.5 \times 10^{7}$ and $1.28 \times 10^{8}$ cells $/ \mathrm{ml}$ for the adeninerequiring and the baker's yeasts, respectively.

\section{RESULTS}

Effect of Methionine on Yeast Growth in the Medium with Fixed Amount of Adenine

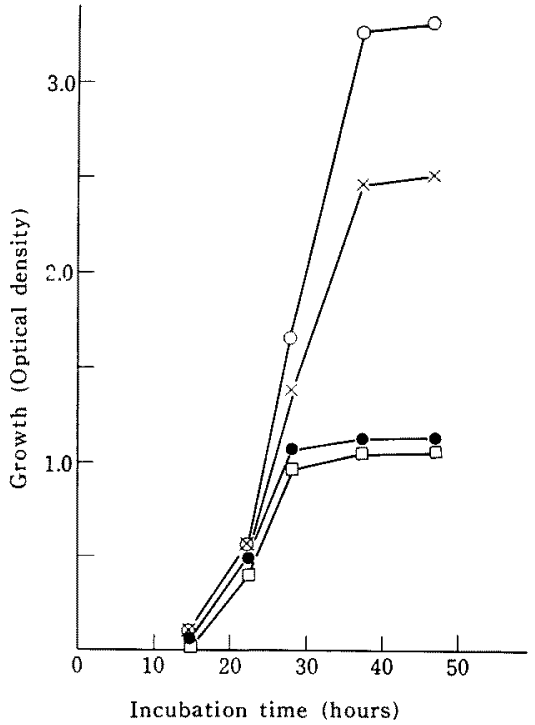

FIG. 1. Effect of Methionine on Growth of the Adenine-Requiring Yeast.

The amount of adenine sulfate added to the media was 0.124 mmoles per liter. The following additions of $\mathrm{DL}$-methionine in $\mathrm{mM}$ were made; $\mathrm{O}: 0$, $x: 1.34,-3.35$ and $\square: 13.40$. The media which contained $6.70,26.80$ and $67.02 \mathrm{mM}$ DLmethionine gave growth curves the same as those in 3.35 or $13.40 \mathrm{mM}$ media.
Fig. 1 shows growth curves of the adeninerequiring yeast cultured with various amounts of methionine but with fixed amount of adenine. Growth of yeast cells was markedly inhibited when methionine, over $3.35 \mathrm{~mm}$, was added to the synthetic medium. Noteworthy was the fact that growth curves were almost identical in the media which contained methionine over $3.35 \mathrm{~mm}$.

In order to know whether or not the inhibition has some relationship with the capacity of adenine biosynthesis, the effect of methionine on growth of the baker's yeast which synthesizes adenine was investigated. Fig. 2 indicates that the pattern of inhibition by methionine was very similar to that of the adenine-requiring mutant. However, higher

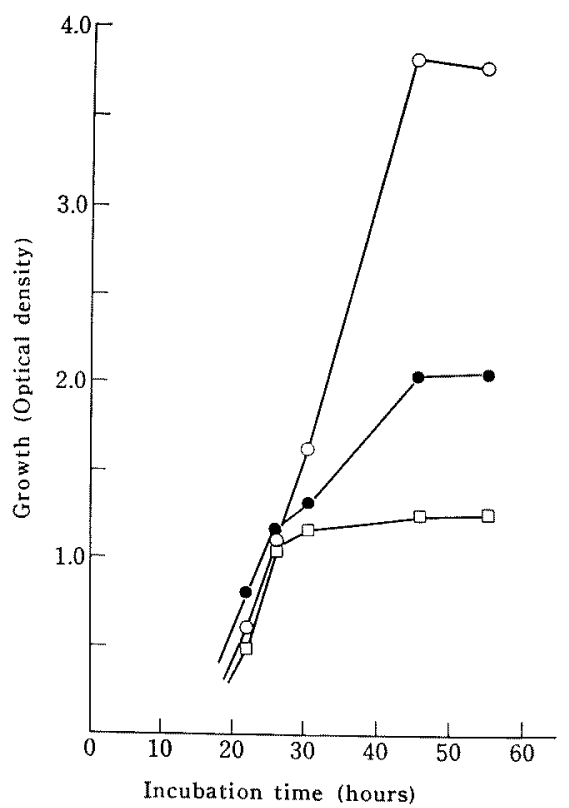

FIG. 2. Effect of Methionine on Growth of the Baker's Yeast.

The following additions of DL-methionine in $\mathrm{MM}$ were made to the synthetic medium; $\bigcirc: 0$, 3.35 and $\square: 13.40$. The medium which contained $67.02 \mathrm{mM}$ DL-methionine gave the similar growth curve as that obtained with the $13.40 \mathrm{~mm}$ medium. 
concentrations of methionine were needed to inhibit the yeast growth, since only a partial inhibition was observed at methionine concentration of $3.35 \mathrm{~mm}$, which was sufficient to inhibit completely the later growth of the adenine-requiring strain after 28 hours.

\section{Competitive Inhibition of Adenine by Methionine} on Growth of Yeast Cells

As shown in Table I, growth of the adeninerequiring yeast incubated in the medium with relatively low concentrations of adenine was markedly inhibited by the addition of methionine, and further supplement of adenine to the medium restored the growth. Although the data presented in Table I were not conclusive, but they suggest the competitive nature of this inhibition.

TABLE I. RELATIONSHIP OF ADENINE AND METHIONINE ON GROWTH OF THE ADENINE-REQUIRING YEAST

\begin{tabular}{clrrrr} 
Methionine mM & \multicolumn{4}{c}{ Growth (O. D. $660 \mathrm{~m} \mu$ ) } \\
\cline { 1 - 1 } Adenine mM & 0 & 3.35 & 14.48 & 67.02 \\
0.0124 & 1.45 & 0.60 & 0.27 & 0.25 \\
0.0371 & 1.66 & 0.70 & 0.59 & 0.55 \\
0.0618 & 2.05 & 0.94 & 0.90 & 0.78 \\
0.124 & 3.41 & 1.31 & 1.08 & 0.97 \\
0.247 & 3.51 & 2.83 & 1.58 & 1.72 \\
0.495 & 3.51 & 3.11 & 3.11 & 2.97
\end{tabular}

The yeast was grown in the medium with various amounts of adenine and methionine for 48 hours.

\section{Reversal of the Methionine Inhibition}

Foregoing experiments suggest that the inhibition was due to the exhaustion of adenine or the decrease of adenine level in the culture medium of the adenine-requiring mutant. In fact, the supplement of adenine to the culture at the time before the inhibition by methione became evident, the inhibition was overcome almost completely (Fig. 3a). The addition of adenine to the medium where the inhibition had already occurred resulted in a partial restoration (Fig. 3b). Although the addition of glucose had little or no effect in both cases, supplements of glucose with adenine markedly stimulated the growth in either case (Fig. 3a and b).

Similar experiment was performed with the baker's yeast. As shown in Fig. 4a, when adenine was added to the medium at the time just before the growth inhibition occurred, the inhibition was partially reversed. The adenine addition, however, had no or

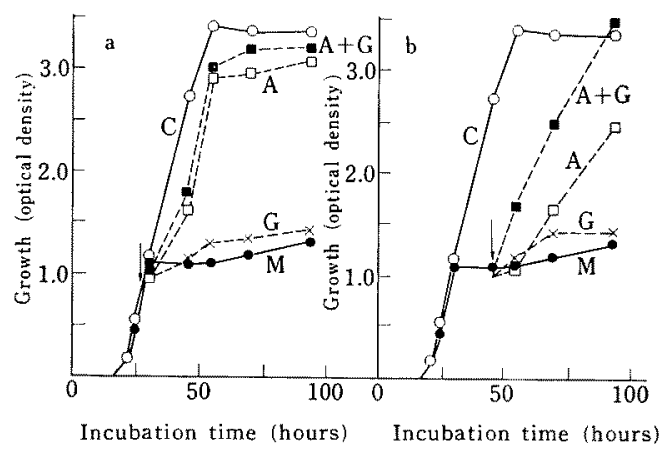

FIG. 3. Reversal of the Methionine Inhibition in the Adenine-Requiring Yeast.

Control cultures $(\mathrm{C})$ were run with methioninefree media. The following additions were done in $\mathrm{mM}$ to the culture medium which contained $13.4 \mathrm{mM}$ of methionine $(M)$ at the time indicated by arrows: 0.495 of adenine sulfate $(A), 50.46$ of glucose $(G)$, and 0.495 of adenine sulfate plus 50.46 of glucose $(A+G)$.

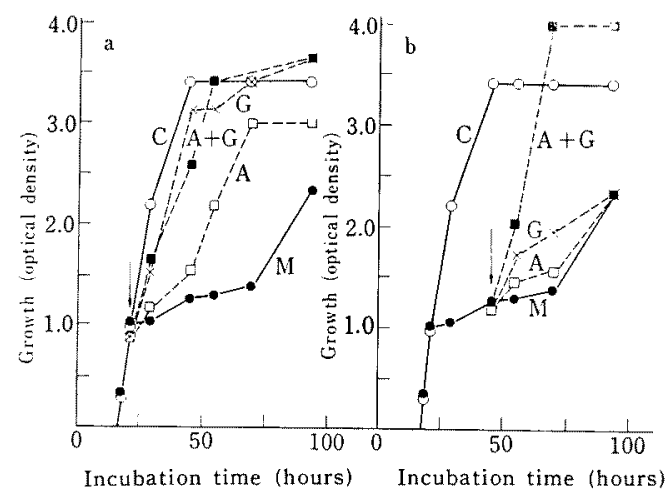

FIG. 4. Reversal of the Methionine Inhibition in the Baker's Yeast.

The conditions were the same as Fig. 3 except that the concentrations of adenine sulfate and glucose employed were $1.24 \mathrm{~mm}$ and $25.23 \mathrm{~mm}$, respectively. 
little effect when it was done at the time when growth was already inhibited (Fig. 4b). The addition of glucose in place of adenine has almost the same or even better effect in restoring growth than adenine (Fig. $4 \mathrm{a}$ and b). Again the addition of both adenine and glucose reversed the inhibition even it was made at the earlier or later stage of growth (Fig. 4a and b). When the baker's yeast was grown in the methionine-containing medium, unlike the adenine-requiring cells, a secondary growth was observed as shown in Fig. $4 a$ and $b$.

\section{DISCUSSION}

The adenine-requiring yeast used in this experiment showed no appreciable growth in an adenine-free medium. However, the addition of a relatively small amount of adenine, for example $2.47 \mu \mathrm{m}$, resulted in a considerable growth $(\mathrm{OD}=1.86)$ by 69 hours culture. The adenine content in yeast cells was roughly calculated from the amount of total nucleic acids in cells grown under the conditions. It was found that the amount of adenine in cells was about ten times as much as that amount of adenine added to the medium. Thus, it is apparent that this mutant has no block in the biochemical sequences leading to adenine biosynthesis. It was supposed that a relatively small amount of adenine might trigger the adenine biosynthesis in this organism, although the precise mechanism has not yet been elucidated.

The growth inhibition of the adenine- requiring strain was an apparent competitive inhibition of adenine by methionine, and either adenine or adenine plus glucose reversed the inhibition in both the mutant and the baker's yeasts. These findings suggest that the growth of yeasts became inhibited as the result of an adenine deficiency in the methionine medium. Since it is already known that a large amount of S-adenosylmethionine is accumulated in yeast cells which were grown in a medium containing methionine, ${ }^{1,21}$ it might be considered that the deficiency in adenine in the yeast cells was caused by Sadenosylmethionine formation which acts as a trap for adenine compounds. Alternatively, methionine might be an inhibitor of adenine biosynthesis, thus, the growth of both yeasts was inhibited by methionine and was restored by adenine supplement. Although DL-methionine was used in the present experiment, the compound which inhibits the yeast growth appears to be L-methionine, since the addition of an equivalent amount of $L$-methionine to the medium resulted in the similar inhibition pattern of the adenine-requiring yeast as that obtained with oL-methionine.

The sensitivity to methionine differs from strain to strain. Growth of yeasts including Candida guilliermondii, Rhodotorula mucilaginosa and Saccharomyces rouxii was not affected by methionine under similar conditions. The result might be explained either by a weaker activity of S-adenosylmethionine formation or a stronger capacity of adenine biosynthesis in these organisms. 